\title{
Suprathermal helium in corotating interaction regions: combined observations from SOHO/CELIAS/STOF and ACE/SWICS
}

\author{
J. Yu ${ }^{1}$, L. Berger ${ }^{1}$, R. Wimmer-Schweingruber ${ }^{1}$, P. Bochsler ${ }^{2}$ B. Klecker ${ }^{3}$, M. Hilchenbach ${ }^{4}$, and R. Kallenbach ${ }^{4}$ \\ 1 Institut für Experimentelle und Angewandte Physik (IEAP), Christian-Albrechts-Universität zu Kiel, Leibnizstrasse 11, 24118 Kiel, \\ Germany \\ e-mail: yu@physik.uni-kiel.de \\ 2 University of Bern, 3012 Bern, Switzerland \\ 3 Max-Planck-Institut für extraterrestrische Physik, Garching, Germany \\ 4 Max-Planck-Institut für Sonnensystemforschung, 37077 Göttingen, Germany
}

Received 5 April 2016 / Accepted 1 November 2016

\begin{abstract}
Context. Energetic particle enhancements that are associated with corotating interaction regions (CIRs) are typically believed to arise from the sunward propagation of particles that are accelerated by CIR-driven shocks beyond $1 \mathrm{AU}$. It is expected that these sunwardtravelling particles will lose energy and scatter, resulting in a turnover of the energy spectra below $\sim 0.5 \mathrm{MeV} /$ nuc. However, the turnover has not been observed so far, suggesting that the CIR-associated low-energy suprathermal ions are accelerated locally close to the observer.

Aims. We investigate the variability of suprathermal particle spectra from CIR to CIR as well as their evolution and variation as the observer moves away from the rear shock or wave.

Methods. Helium data in the suprathermal energy range from the Solar and Heliospheric Observatory/Charge, Element, and Isotope Analysis System/Suprathermal Time-of-Flight (SOHO/CELIAS/STOF) were used for the spectral analysis and were combined with data from the Advanced Composition Explorer/ Solar Wind Ion Composition Spectrometer (ACE/SWICS) in the solar wind energies. Results. We investigated sixteen events: nine clean CIR events, three CIR events with possible contamination from upstream ion events or solar energetic particles (SEPs), and four events that occurred during CIR periods that were dominated by SEPs. Six of the nine clean CIR events showed possible signs of a turnover between $\sim 10-40 \mathrm{keV} /$ nuc in the fast solar wind that trails the compression regions. Three of them even showed this behaviour inside the compressed fast wind. The turnover part of the spectra became flatter and shifted from lower to higher energies with increasing connection distance to the reverse shock. The remaining three clean events showed continuous power-law spectra in both the compressed fast wind and fast wind regions, that is, the same behaviour as reported from previous observations. The spectra of the seven remaining events are more variable, that is, they show power law, turnover, and a superposition of these two shapes.
\end{abstract}

Key words. acceleration of particles - shock waves - solar wind - Sun: heliosphere - Sun: rotation

\section{Introduction}

Corotating interaction regions (CIRs) commonly form when high-speed solar wind streams that emerge from solar coronal holes overtake low-speed solar wind streams that have been emitted earlier (Gosling et al. 1978). The interaction between these two stream plasmas creates a compression region that corotates with the Sun and bounds with the leading and trailing edge. Forward and reverse shocks can form at the leading and trailing edge, respectively, when the interaction strengthens enough, normally at heliocentric distances greater than 2 AU (Balogh et al. 2000). It is widely known that suprathermal particles observed in association with CIRs have been interpreted to be accelerated in the compressed plasmas and shocks (Fisk \& Lee 1980; Schwadron et al. 1996). During solar minimum, when the coronal mass ejections (CMEs) and solar flares are rare, CIRs are the dominant source of suprathermal particles in the interplanetary medium at $1 \mathrm{AU}$ (Fisk \& Jokipii 1999). Investigations of the origin of CIR-associated suprathermal particles have shown that these particles appear not only inside CIRs, but also often persist within the leading parts of fast-wind regions (Reames et al. 1997; Mason et al. 1997; Simnett et al. 1998; Hilchenbach et al. 1999; Chotoo et al. 2000). Commonly there are believed to be two origins: (1) local acceleration by adiabatic compression in compressive turbulence (Fisk \& Gloeckler 2006, 2007; Fisk et al. 2010), from transit-time damping of magnitude variations in the magnetic field (Schwadron et al. 1996); and (2) sunward-directed particles accelerated at remote reverse shocks as described by the model of Fisk \& Lee (1980) (in this paper, we call this "remote acceleration"). Local acceleration is ubiquitous in the solar wind, and in quiet-time conditions, suprathermal tails show a common energy-spectrum shape: a power law with a constant index of -1.5 (Fisk \& Gloeckler 2006). For particles accelerated remotely (origin 2), velocity distribution functions have been theoretically discussed by Fisk \& Lee (1980), who considered a model of diffusive acceleration at CIR shocks several AU away followed by propagation into the inner heliosphere along magnetic field lines, including adiabatic cooling. The spectral shape of these sunwardstreaming particles should be a power law multiplied with an exponential rollover, when the particle velocity is much higher than the speed of the corotating frame. Fisk \& Lee (1980) pointed out that these accelerated sunward particles should have a lowenergy threshold well above solar wind energies, as they have had multiple interactions with the shock and must have had sufficient speed after their first interactions to propagate upstream 


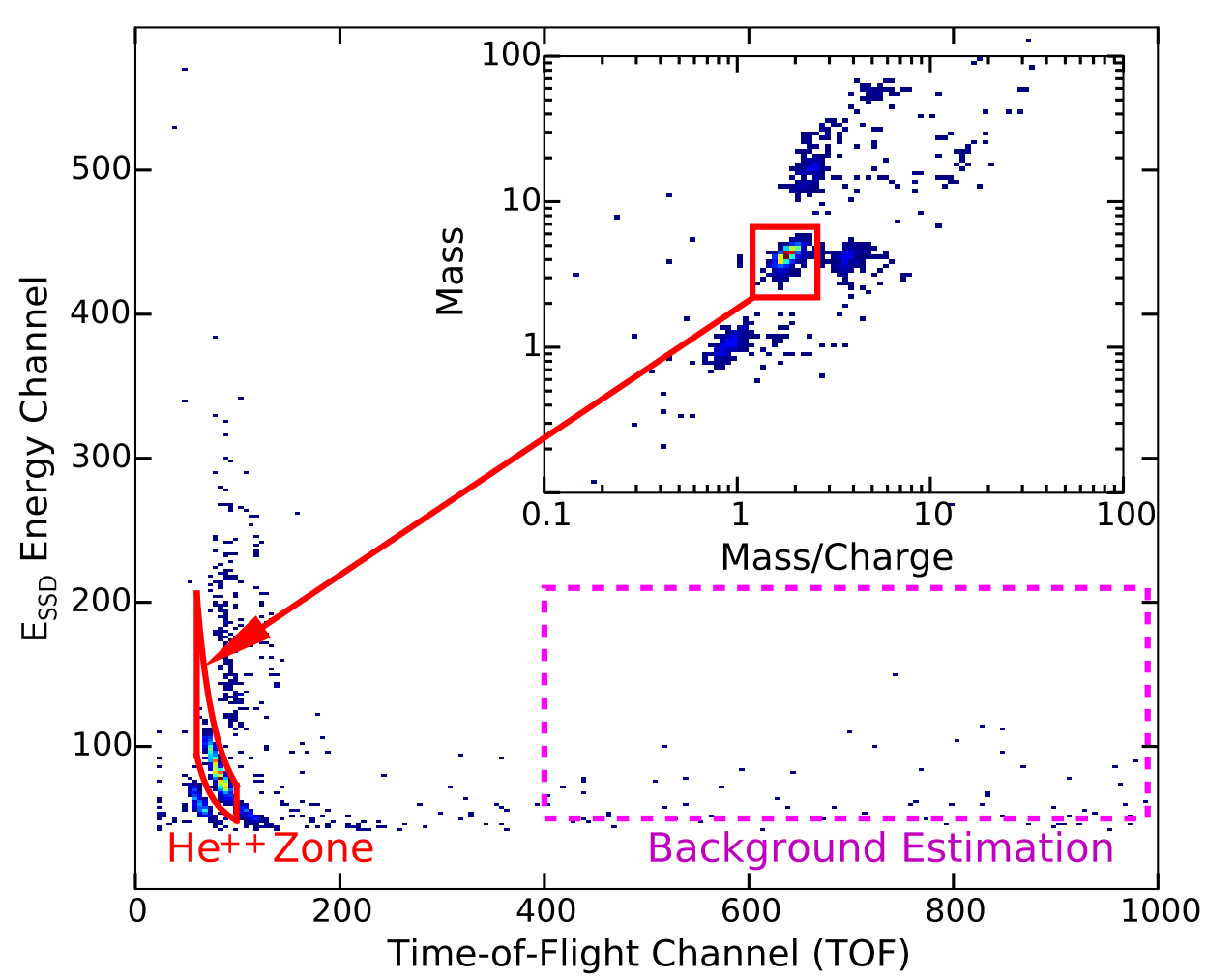

Fig. 1. STOF data acquired for the range of 60-84 E/q steps during days 206-211 in 2003. The panel shows the measured energy, $E_{\mathrm{SSD}}$ vs. TOF, while its inset shows the same data mapped to $m-m / q$ space. Alpha particles are surrounded by a red square in the inset. The corresponding $E_{\mathrm{SSD}}-\mathrm{TOF}$ measurements are surrounded by a mapped closed red curve that corresponds to the red rectangle in $m-m / q$ space. The accidental coincidence events (or instrumental background) are uniformly distributed throughout the entire TOF region and fall randomly within the real ion zone, e.g., $\mathrm{He}^{++}$. The large dashed magenta rectangle in the high-TOF region is used to estimate the background level of our measurements.

in the solar wind. In other words, the energy spectra may exhibit a turnover above solar wind energies. However, Chotoo et al. (2000), Mason et al. (1997), Reames et al. (1997) did not observe this turnover. CIR events studied by them showed powerlaw spectra above solar wind energies in both compressed fastwind region and fast-wind region.

We used data from Solar Wind Ion Composition Spectrometer (SWICS) and Suprathermal Time-of-Flight (STOF) to study the temporal evolution of the alpha-particle spectra from the solar wind through the suprathermal energy range in the 16 events given in Table 1 . We found that 6 of 9 clean CIR events are likely to show a turnover in their spectra between $\sim 10$ and $40 \mathrm{keV} /$ nuc.

\section{Instrumentation}

The STOF mass spectrometer, which is part of the Charge, Element, and Isotope Analysis System (CELIAS) on board the Solar and Heliospheric Observatory (SOHO), measures suprathermal ions (Hovestadt et al. 1995). An electrostatic deflection system covers $35-660 \mathrm{keV} / \mathrm{Q}$ in 120 logarithmically spaced voltage steps. The geometrical factor of STOF is $0.05 \mathrm{~cm}^{2} \mathrm{sr}$ (Bamert et al. 2002) with the field of view $\left(3^{\circ} \times 17^{\circ}\right)$, pointing $7^{\circ}$ west off the SOHO-Sun line (Hilchenbach et al. 2001). The Proton Monitor (PM) of CELIAS consists of a Wide Angle, Variable Energy/charge (WAVE) passband deflection system to measure the solar wind proton bulk speed, density, thermal speed, and north/south flow direction (Ipavich et al. 1998). A detailed description of the CELIAS instruments has been given by Hovestadt et al. (1995). The SWICS on board the Advanced Composition Explorer (ACE) was designed to measure the composition of the solar wind and pickup ions from $\sim 0.6$ to $\sim 86 \mathrm{keV} / \mathrm{Q}$ (see details in Gloeckler et al. 1998; Berger 2008). The Solar Wind Electron, Proton, and Alpha Monitor (SWEPAM) of ACE measures the solar wind plasma, electron, and ion fluxes as functions of their direction and energy. These data provide detailed knowledge of the physical parameters of the solar wind in every minute (see details in McComas et al. 1998), which were used to compare with the measurement of PM in this paper. The Magnetometer (MAG) on ACE measures the local interplanetary magnetic field (IMF) direction and magnitude to establish the large-scale structure and fluctuation characteristics of the IMF (see details in Smith et al. 1998).

STOF uses a triple-coincidence technique to identify particles; a start and stop trigger are generated in the time-offlight (TOF) unit, the third comes from silicon solid-state detectors (SSDs). The measured TOF and energy, together with the known energy per charge of the particles (as determined by the electrostatic entrance system), allows us to measure the energy, mass, and mass per charge of a suprathermal ion (see e.g. Bamert et al. 2002). The triple coincidence suppresses accidental coincidences of penetrating energetic particles. However, using instrumental house-keeping data, we found that the count rate of the start and stop triggers of the micro channel plates (MCPs) on the TOF unit is correlated with the flux of solar ultraviolet light (UV). A possible leakage of photons may occur at the entrance of STOF, as the instrument faces the Sun almost directly. Then MCPs are triggered by a high flux of photoelectrons, resulting in much higher background than originally anticipated (Hilchenbach et al. 1998).

Figure 1 shows data acquired with STOF. The graphic shows the measured energy, $E_{\mathrm{SSD}}$, vs. TOF, with an inset showing the corresponding STOF data mapped to mass/mass per charge 
space. Five types of ions can readily be distinguished in the inset. From bottom to top they are protons, alpha particles (surrounded by a red rectangle), $\mathrm{He}^{+}$, a $\mathrm{CNO}$ group, and heavy ions such as $\mathrm{Mg}, \mathrm{Si}$, and iron ions. The corresponding $E_{\mathrm{SSD}}$ vs. TOF is shown in the surrounding plot, and the alpha-particle track is the region enclosed in the red line. To estimate the effect of the background on the $\mathrm{He}^{++}$measurements, we used a method very similar to the one used by Hilchenbach et al. (1998) for HSTOF. We have verified that the background to the right of the $\mathrm{He}^{++}$particles is evenly distributed in TOF. Since the background is caused by UV-triggered accidental coincidence events, these events should scatter uniformly throughout the entire TOF region, mingled with the real events. Thus, we can sample the background at higher TOF (where there are no heavy ions) to obtain a robust estimate of the expected background around the alpha particles. This is indicated by the dashed magenta rectangle. All events falling within any energy channel in this rectangle provide a true measure of the background in the same energy channel intervals in the real ion track. Then we can well estimate the background level in all the energy channels covered by the red $\mathrm{He}^{++}$zone.

A further instrumental effect that needs to be accounted for is the gradual and unknown degradation of the MCPs, which means that we do not know the absolute fluxes measured with this instrument. Following Bamert et al. (2002), we therefore used arbitrary units for the differential fluxes described in this paper. Fortunately, the MCP degradation during CIR epochs is slow, so that we assume constant MCP efficiency during each CIR event in this paper.

\section{Observations}

Figure 2 shows solar wind plasma and magnetic field measurements for a CIR that occurred between July 26 and 27, 2003 (days of year 207-208). Following Chotoo et al. (2000), Richardson et al. (1993), we marked four regions in the plot: the slow wind region $(S)$, the compressed slow wind region $\left(S^{\prime}\right)$, the compressed fast wind region $\left(\mathrm{F}^{\prime}\right)$, and the fast wind itself $(F)$. Throughout four regions, the mean charge states of iron measured by ACE/SWICS lies around $11^{+}$, consistent with typical values in the solar wind (Lepri et al. 2001). The stream interface $\left(\mathrm{S}^{\prime}-\mathrm{F}^{\prime}\right)$ is indicated by the vertical line in Fig. 2 and is characterized by a drop of the $\mathrm{O}^{7+} / \mathrm{O}^{6+}$ abundance ratio measured with SWICS in the bulk solar wind (Wimmer-Schweingruber et al. 1997, 1999). The leading (S-S') and trailing edge $\left(\mathrm{F}^{\prime}-\mathrm{F}\right)$ of the CIR were determined by the total pressure (Jian et al. 2006). Bučík et al. (2009) found that CIR boundaries can be well defined when the total pressure exceeds $50 \mathrm{pPa}$ (indicated by the horizontal dashed line in Fig. 2), which is slightly higher than that in the background solar wind, which typically is $20-30 \mathrm{pPa}$, according to Jian et al. (2006). The total pressure $P$ was obtained from the sum of plasma and magnetic field pressure, that is, $P=n_{\mathrm{p}} v_{\mathrm{th}}^{2} m+B^{2} / 2 \mu_{0}$, where $n_{\mathrm{p}}$ and $v_{\text {th }}$ are the proton density and thermal speed, respectively, and $B$ is the magnitude of the magnetic field. Because SOHO has no magnetometer, we used magnetic field data from ACE/MAG (which is also around L1). Comparing plasma parameters (bulk speed, thermal speed, and proton density) measured by PM with those of SWEPAM, we see that the physical conditions at SOHO and ACE were almost the same, and that the time difference between passages of the CIR boundaries is less than ten minutes. The CIR shown in Fig. 2 was bounded by a reverse shock (vertical line separating $\mathrm{F}^{\prime}$ from $\mathrm{F}$ ). We clearly see that the suprathermal $\mathrm{He}^{++}$intensity peaks inside the decelerated and compressed fast-wind region $\left(\mathrm{F}^{\prime}\right)$, close to the reverse shock. In contrast,
CIR 04

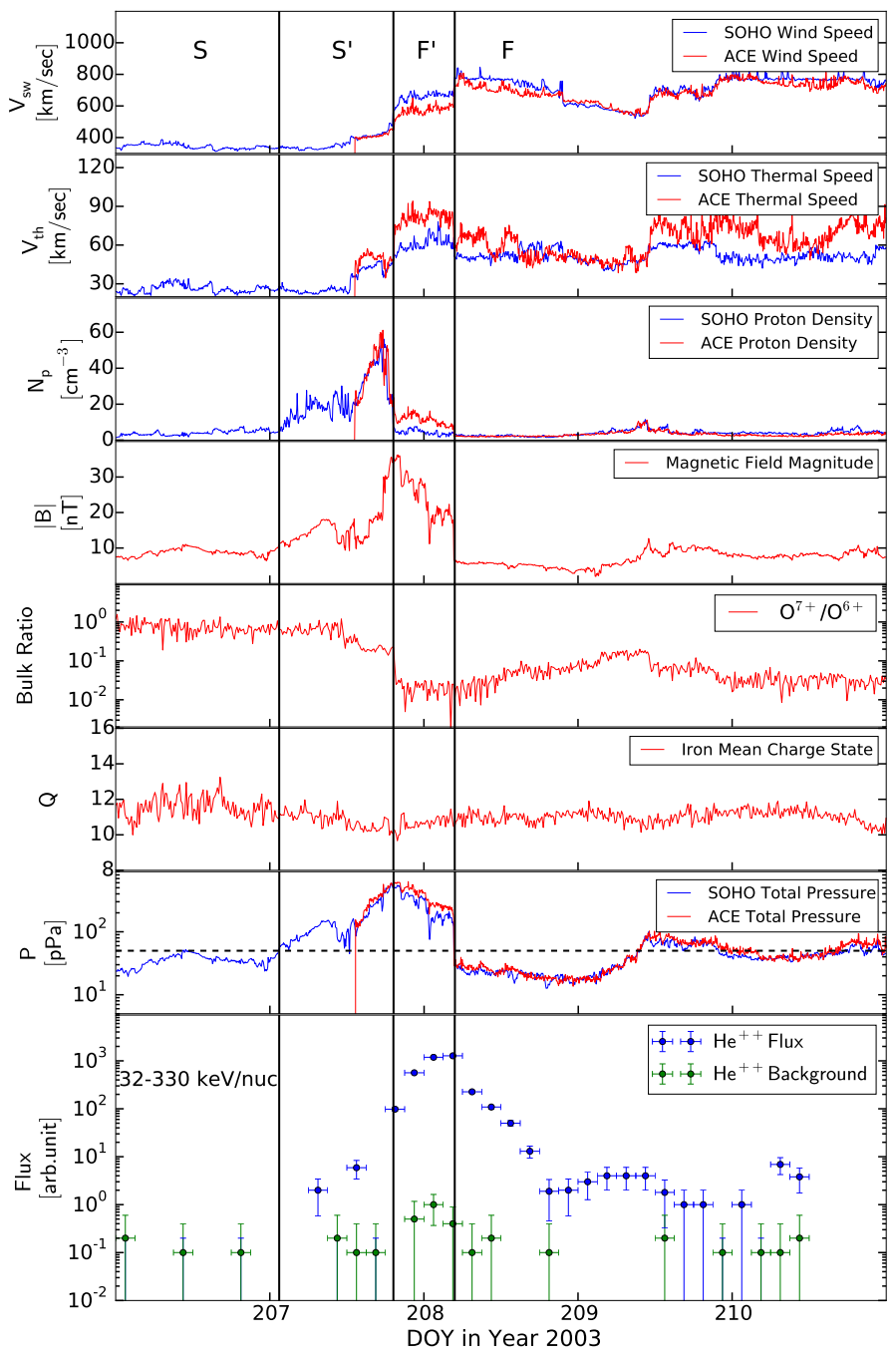

Fig. 2. Time profiles of CIR-associated suprathermal helium enhancement identified by STOF during the period from DOY 206 to DOY 211, 2003. Shown from top to bottom are the 5-min averaged solar wind proton speed, the thermal speed, and the proton density measured by PM and SWEPAM, the 4-min averaged interplanetary magnetic field strength measured by ACE/MAG, the 12 -min averaged $\mathrm{O}^{7+} / \mathrm{O}^{6+}$ abundance ratio measured by ACE/SWICS, the 12-min mean charge state of iron measured by ACE/SWICS, the total pressure $\left(n_{\mathrm{p}} v_{\mathrm{th}}^{2} m+B^{2} / 2 \mu_{0}\right)$, and three-hour-averaged helium fluxes and the corresponding background estimate from $\sim 32$ to $330 \mathrm{keV} /$ nuc.

suprathermal particles are very rare in the $S$ and $S^{\prime}$ regions. After passage of the reverse shock, suprathermal particles continue to be observable for more than one day. They are commonly believed to be the sunward particles accelerated by the reverse shock far beyond the Earth orbit. In other words, the observer saw the duration of the CIR particle event, which was longer than that of the CIR compression region itself. The background level shown in green was estimated using the method described above. The signal-to-noise ratio $(\mathrm{S} / \mathrm{N})$ in the $\mathrm{F}$ and $\mathrm{F}^{\prime}$ regions is higher than 100 , confirming that our observations are due to real $\mathrm{He}^{++}$particles.

\subsection{Event selection}

Table 1 lists the sixteen CIR events investigated in this paper. We limited our investigation to the time interval from February 1999 
Table 1. CIR events with suprathermal ions at STOF.

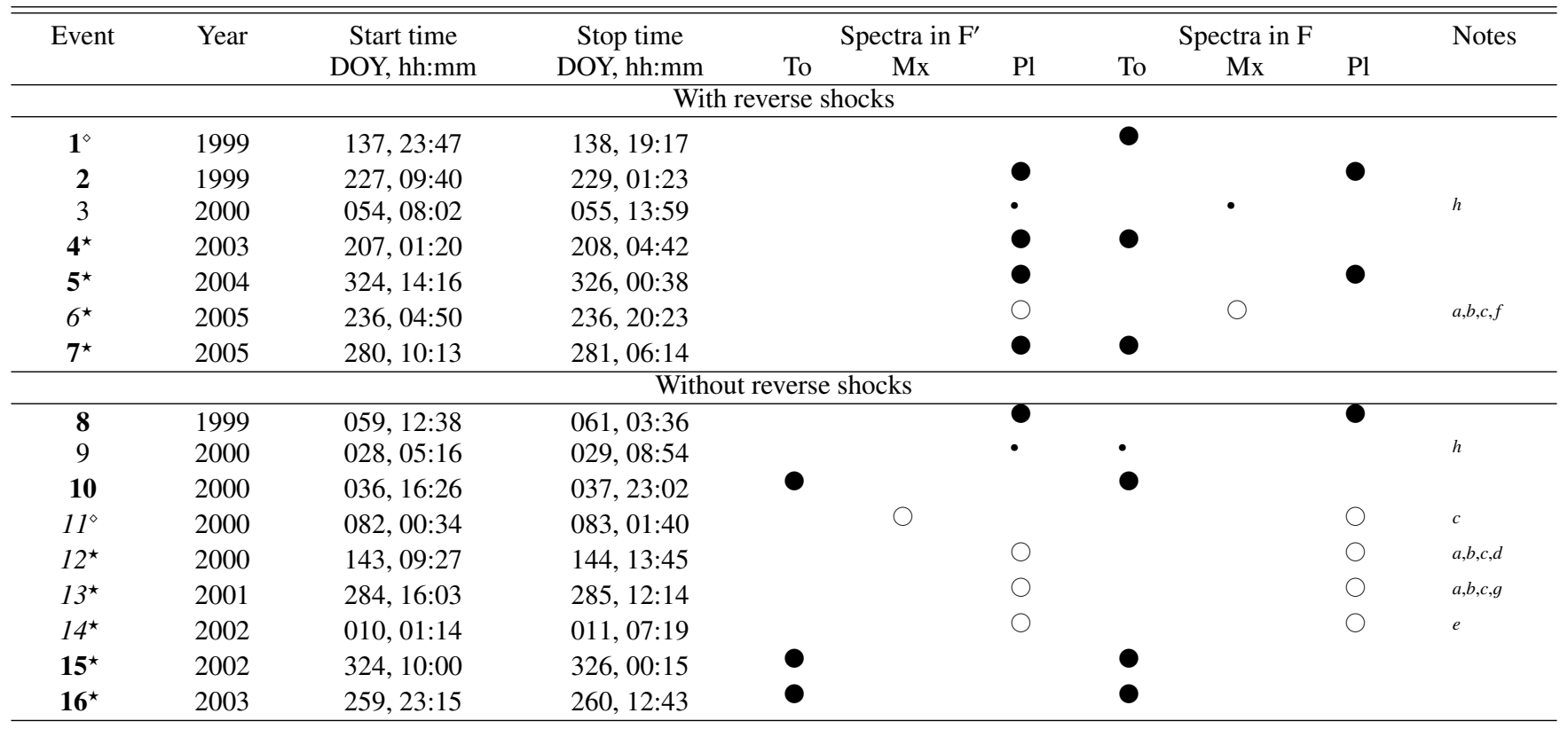

Notes. Start and stop time of the compression region based on the physical properties of the solar wind are shown with day of year and coordinated universal time (DOY, hh:mm in the table). "To", "Mx" and "Pl" columns list different spectral shapes: turnover spectra, the superposition of turnover and power-law spectra, and power-law spectra, respectively. $\left.{ }^{(}\right)$Events 1 and 11 have a data gap in STOF measurements and do not cover the full time period. $\left.{ }^{\star}\right)$ Events 4-7 and 12-16 include SWICS measurements. $\left.{ }^{(}\right)$Means "clean" CIR events, listed in Jian et al. (2006) and Mason et al. (2008). ${ }^{\left({ }^{(}\right)}$Indicates ICMEs or SEPs. ${ }^{(\bullet)}$ Marks CIR events that are possibly contaminated by upstream ion events. ${ }^{(a)}$ Interacted with ICMEs, suggested by Jian et al. (2006). ${ }^{(b)}$ In the ICME list surveyed by Richardson \& Cane (2010). ${ }^{(c)}$ In the SEP list studied by Cane et al. (2010). ${ }^{(d)}$ Analysed by Mason et al. (2004) as ${ }^{3}$ He-rich SEP events. ${ }^{(e)}$ Included in the list of large SEP events, given by Desai et al. (2006). ${ }^{(f)}$ Can be found in the SEP list of Cliver et al. (2012). ${ }^{(g)}$ In the interplanetary shock event list of Desai et al. (2003). ${ }^{(h)}$ Marks CIR events that are possibly contaminated by upstream ion events. There are strong indications of upstream events associated with CIR 09. For the case of CIR 03, however, upstream events are not clear (suggested by A. Klassen, pers. comm.).

to October 2005, when STOF (and especially its MCPs) performed well and the surveyed CIR events had good ion counting statistics. Based on the CIR list from Jian et al. (2006), we selected sixteen particle events for our study, the S/Ns of which are all better than 10. Twelve events are CIR-associated events (Mason et al. 2008), and 4 are solar energetic particle (SEP) events (Mason et al. 2004; Desai et al. 2006; Cane et al. 2010). As shown in Table 1, we divided our study into two groups: seven events bounded with reverse shocks, and nine events with a gradual change at the trailing edge. We added ACE/SWICS data for events occurring after May 2000 (indicated with ${ }^{\star}$ in Table 1), thus extending the coverage to lower energies. This resulted in energy spectra ranging from the solar wind bulk distribution to suprathermal tails. STOF data for events 1 and 11 (marked with $^{\diamond}$ ) do not cover the full time period. Out of the 12 CIR events, the 9 without notes are considered as clean events, all of which have the classic CIR structures similar to Fig. 2 (see event numbers with bold font in Table 1). For CIR 3 and 9 (see normal-font event numbers in Table 1), we found hints of upstream ion events following these 2 events (A. Klassen, pers. comm.). Although event 11 was included in the CIR event list of Mason et al. (2008), it was considered as an SEP event by Cane et al. (2010). Here, we treat it as a contaminated event. We have identified complex CIR structures during 4 SEP events in this work, see one example in Fig. 3. We can only determine the boundaries of the compression region but not the stream interface for this event. The variations in the $\mathrm{O}^{7+} / \mathrm{O}^{6+}$ ratio are not consistent with a stream interface being present in this "CIR" (Wimmer-Schweingruber et al. 1997, 1999). Moreover, the mean charge state of iron abruptly jumps from $\sim 10^{+}$to $\sim 15^{+}$between $16: 21$ on day 284 and 12:36 on day 285, which indicates an ICME event according to Lepri et al. (2001), Lepri \& Zurbuchen (2004), consistent with previous observations (Jian et al. 2006; Richardson \& Cane 2010). Spectral shapes of all events marked with a filled and empty large circle, and a filled small circle in $\mathrm{F}$ and $\mathrm{F}^{\prime}$ regions are discussed in detail in the following sections.

\subsection{Clean events with reverse shocks}

Based on Fisk \& Lee (1980), the velocity distribution function or differential flux of suprathermal particles is a function of heliocentric distance between the observer and the shock. We here computed the radial distance from the spacecraft at $1 \mathrm{AU}$ to the location of the reverse shock, according to Morris et al. (2001). The equation for the connection distance is $l=v_{\mathrm{f}} \cdot v_{0} \cdot \Delta t /\left(v_{\mathrm{f}}-v_{0}\right)$, where $v_{\mathrm{f}}$ is the local solar wind speed in the $\mathrm{F}$ region, $v_{0}$ is the solar wind speed just ahead of the reverse shock, and $\Delta t$ is the time after passing the reverse shock. Figure 4 shows the energy spectrum of the first CIR event in Table 1 in the undisturbed fastwind region. Because of a data gap, we have no $\mathrm{He}^{++}$data inside this CIR and for the time corresponding to a connection distance out to 4 AU. Following Bamert et al. (2002), we plot fluxes in 12 energy channels (from 32 to $330 \mathrm{keV} / \mathrm{nuc}$ ), each of which sums over $8 E / q$ channels of STOF. We show the estimated 
CIR 13 (SEP)

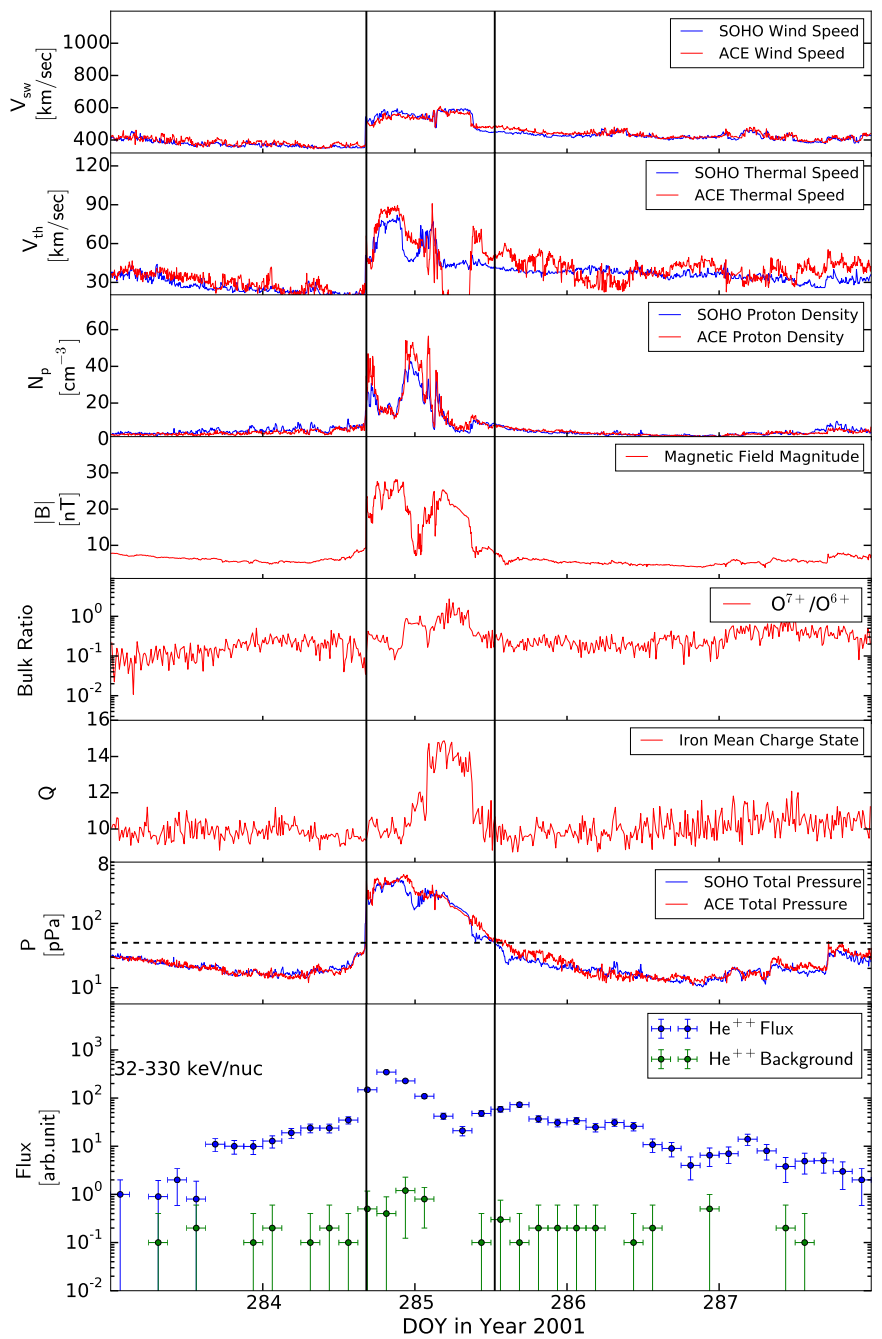

Fig. 3. Same format as Fig. 2, but the event contaminated with an ICME.

one-count level in the plot to evaluate the statistical significance of our measurement. The larger the difference between flux and the one-count level, the more reliable the statistics. Clearly, the spectrum in Fig. 4 is not a power law at low energies, but shows a turnover around $\sim 50 \mathrm{keV} /$ nuc.

Figure 5 shows the alpha-particle spectra for the other six shock-bounded events in four subplots each. From left to right, the subplots illustrate the spectral evolution from the $\mathrm{F}^{\prime}$ region to the $\mathrm{F}$ region. Considering that the $\mathrm{He}^{++}$flux peaks in the $\mathrm{F}^{\prime}$ region and close to the reverse shock, we defined the data from six hours before the reverse shock as a sample of the $\mathrm{F}^{\prime}$ region. As the compression region moves away, the flux of suprathermal particles decreases with time, and different events have different durations, during which the suprathermal particles satisfy our selection criterion $(S / N>10)$. Because of this decrease, we used progressively longer time intervals to obtain a comparable counting statistics in three plots of the F region. Each event has its own one-count level, which was determined using the relation of the differential flux $\mathrm{d} J / \mathrm{d} E$ to count rates,

$\mathrm{d} J / \mathrm{d} E=\frac{c}{g \cdot \Delta E \cdot \Delta t \cdot \eta}$,

where $g$ is the geometric factor, $\Delta E=q \cdot \Delta E / q$, where $\Delta E / q$ is the $E / q$ passband of the electrostatic analyser, $\Delta t$ the accumulation time, and $\eta$ is an unknown efficiency for STOF (it is known

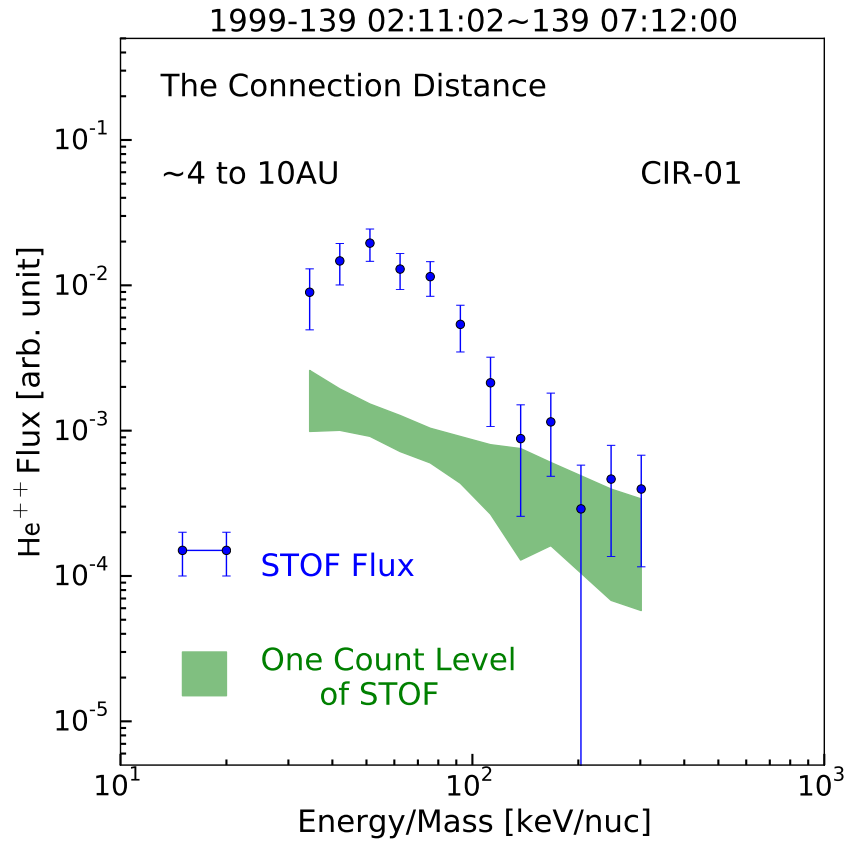

Fig. 4. Energy spectrum after CIR 01 during 02:11 to 07:12 in DOY 139, 1999. One count level is calculated using the flux with an error divided with the raw count in the corresponding energy bin. The connection distance is from the spacecraft to the reverse shock.

for SWICS, however). The one-count level is now determined by the ratio of $\mathrm{d} J / \mathrm{d} E$ to the number of measured counts, $c$. This is unambiguous for SWICS, and requires normalization for STOF to account for the unknown $\eta_{\mathrm{STOF}}$. This normalization is discussed below. We used the same one-count level, but corrected for differing accumulation times across the full compression region and trailing parts. This assumes that the unknown $\eta_{\mathrm{STOF}}$ does not change appreciably during the event. Referring to the house-keeping data of STOF, the efficiency decrease in MCPs over a few days is negligible, therefore we assumed a constant instrumental efficiency for each individual event. For events 04 to 07, we added the spectra from SWICS shown in red in Fig. 5. A quantitative comparison of the STOF and SWICS fluxes is probably impossible and certainly beyond the scope of this paper, especially because of the unknown absolute efficiencies of the MCPs. In addition, SWICS has a much larger field of view than STOF (partially to accommodate the changing ACE pointing and spin). STOF, being on the three-axis-stabilized SOHO, covers a much smaller solid angle. We lined up the SWICS and STOF data by applying a calibration factor for each event. This calibration factor was determined for the spectra in the $\mathrm{F}^{\prime}$ region (left-hand panels) using the counts in the four overlapping energy bins (between the two vertical lines at 32-44 keV/nuc) and by assuming that the suprathermal particles are isotropic. The same calibration factor was used for the three right-hand panels.

Here, we analyse five clean CIR events of the first event group (top half of Table 1). The spectra shown in the leftmost column of Fig. 5 were all accumulated in the $\mathrm{F}^{\prime}$ region and exhibit $E^{-\gamma}$ or $E^{-\gamma} \cdot \exp \left(E / E_{0}\right)$ suprathermal tails above the solar bulk distribution. This spectral shape can be explained by diffusive shock acceleration, consistent with previous research (Fisk \& Lee 1980; Savopulos et al. 1995; Chotoo et al. 2000; Mason et al. 1997). The three right-hand panels show the behaviour in the $\mathrm{F}$ region at successively larger distances from the $\mathrm{F}^{\prime}-\mathrm{F}$ interface. They are marked by their inferred connection distance to the reverse shock in the upper right corner. CIR 02 
A\&A 599, A13 (2017)
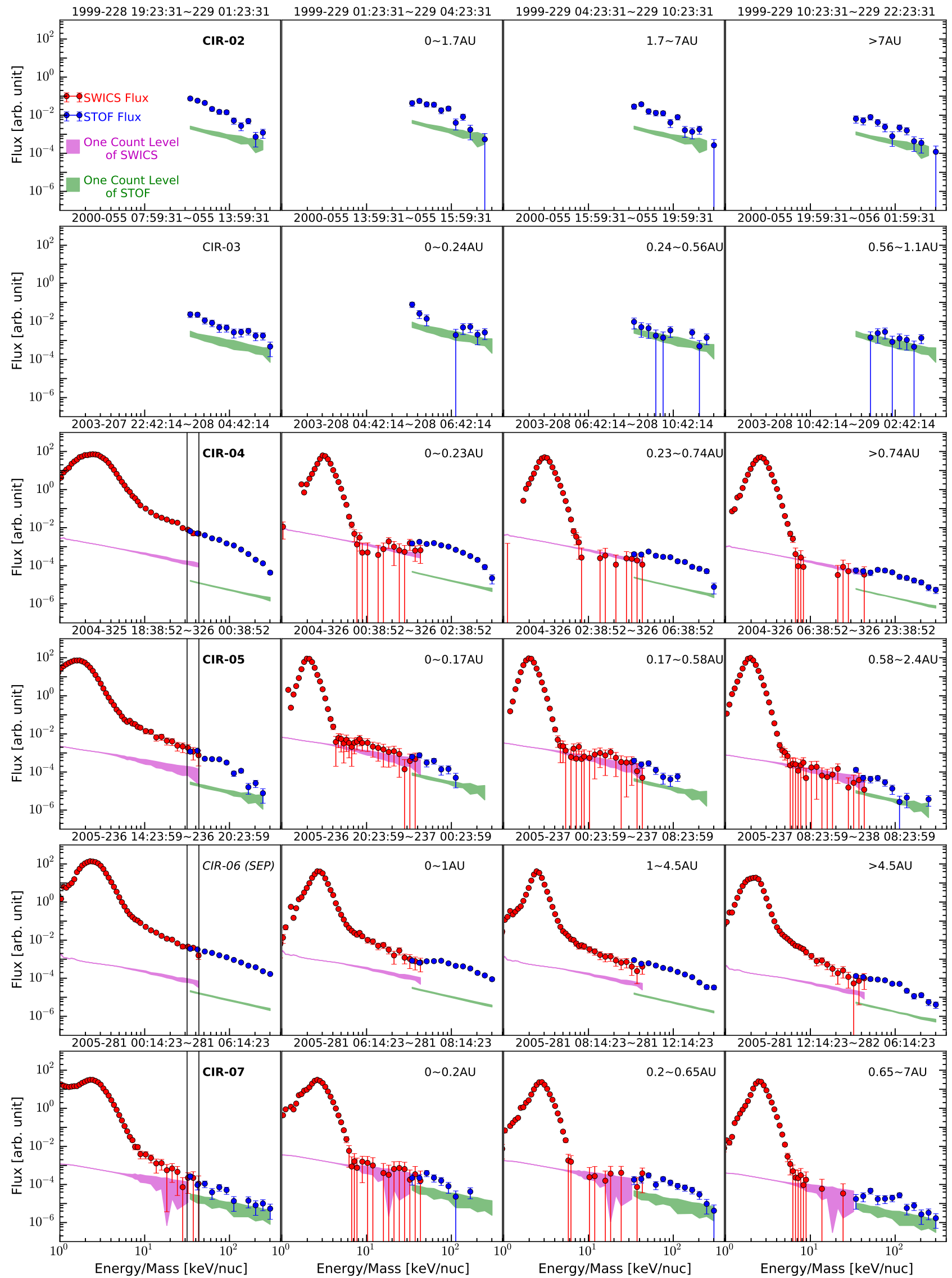

Fig. 5. Two vertical black lines in some plots mark the overlapping energy range between STOF and SWICS. Values within the two lines are used to intercalibrate SWICS and STOF. The first column of the plots displays the spectra inside the compression regions with the event number in the upper right corner. The remaining three columns of the plots show the spectra in the F regions with the connection distance in the upper right corner. 
shows similar spectral shapes in the $\mathrm{F}$ region as in $\mathrm{F}^{\prime}$. Three spectra exhibit power-law tails below around $100 \mathrm{keV} / \mathrm{nuc}$, and bend over above this energy. However, the signature is not very clear in the rightmost plot, possibly because of the statistical fluctuations. In CIR 05, fluxes of suprathermal particles measured by SWICS are only slightly above or at the one-count level. They clearly illustrate continuous power-law spectra from $\sim 5$ to $\sim 100 \mathrm{keV} /$ nuc. Below $\sim 100 \mathrm{keV} / \mathrm{nuc}$, STOF measurements of this event are above the one-count level, while beyond $\sim 100 \mathrm{keV} /$ nuc they indicate a possible exponential decay. CIRs 04 and 07 clearly show a turnover. In three plots in the $\mathrm{F}$ region of CIR 07, all the STOF spectra show a possible turnover at around 32-44 keV. Although SWICS spectra show some suprathermal particles in the second plot, the fluxes are at the one-count level and the errors of these measurement are large. Between 0.2 and $0.65 \mathrm{AU}$, only the one SWICS data point at the highest $E / q$ channel is not an upper limit and fits in well with the STOF data. The rightmost panel shows clearer evidence of the turnover. The only two data points from SWICS are only upper limits, indicating a spectral gap in the flux of SWICS and of STOF, which is consistent with the turnover spectral shape from STOF. More apparent turnover spectra were observed in CIR 04, which was reported by Yu et al. (2016). In the second plot (0-0.23 AU), the flux measured by SWICS between solar wind bulk and $\sim 18 \mathrm{keV} /$ nuc is only an upper limit (below the one-count level), and data that are not upper limits only appear in the higher (SWICS) energy range. STOF data show good counting statistics, and therefore the spectrum probably turns over between $\sim 18$ and $44 \mathrm{keV} /$ nuc. The turnover behaviour continues in the following two plots. In detail, all the suprathermal particles from SWICS stay in or below the one-count level with large errors in the third plot, which might mean that the turnover shifts to the higher energy range covered by STOF. In the fourth plot we observe a spectral gap in the suprathermal flux from SWICS extending from just above the solar wind bulk out to $\sim 20 \mathrm{keV} / \mathrm{nuc}$; this lasted sixteen hours. On the other hand, the STOF spectrum is nearly flat below $\sim 60 \mathrm{keV} /$ nuc and evolves into a power law at higher energies. In summary, in this first group of five clean CIR events with reverse shocks, we found three CIR events $(01$, 04, and 07) that indicate turnover spectra in the F regions above solar wind energies to $\sim 40 \mathrm{keV} /$ nuc. Two remaining CIR events (02 and 05) have continuous power-law spectra.

As we show in Fig. 6, we fitted the spectra of CIR 04 with the following theoretical expressions (2) (downstream of the shock) and (3) (upstream of the shock) given by Fisk \& Lee (1980), as this event is associated with clear turnover spectra in the fastwind region,

$f=\left(\frac{r}{r_{s}}\right)^{-2 /(1-\beta)} v^{-3 /(1-\beta)} \exp \left[-\frac{6 \kappa_{0} \beta v}{V(1-\beta)^{2}}\left(\frac{r}{r_{s}}\right)^{2 / 3}\right]$

$f=\left(\frac{r}{r_{s}}\right)^{2 \beta /(1-\beta)+V /\left(\kappa_{0} v\right)} v^{-3 /(1-\beta)} \exp \left[-\frac{6 \kappa_{0} \beta v}{V(1-\beta)^{2}}\right]$,

where $f$ is the velocity distribution function, $v$ is the ion speed, $r$ is the heliocentric distance of the observer, $r_{s}$ is the heliocentric distance of the reverse shock, $V$ is the solar wind speed, $\kappa_{0}$ is a constant $\left(\kappa_{0}=\kappa / v r\right.$, where $\kappa$ is the diffusion coefficient), and $\beta$ is the inverse of compression ratio at the CIR-driven shock.

First, we fit the spectrum (blue dots) in the $\mathrm{F}^{\prime}$ regions with Eq. (2), assuming $r_{s}=r$. Referring to the approach of Chotoo et al. (2000), we determined the parameters $\beta=0.251$ and $V=787 \mathrm{~km} \mathrm{~s}-1$ directly from measurements of ACE/MAG

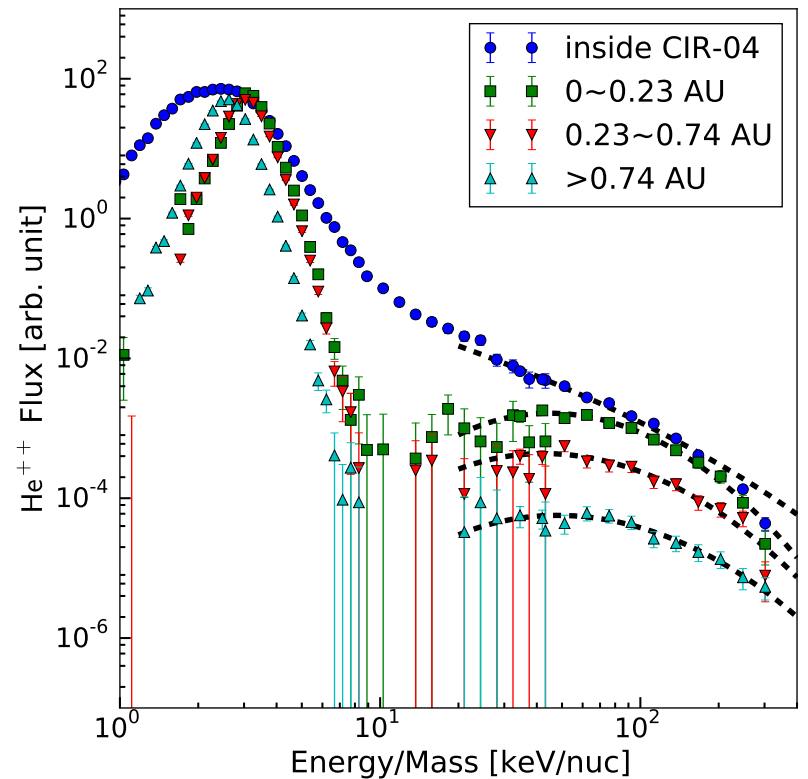

Fig. 6. Energy spectrum of $\mathrm{He}^{++}$in the $\mathrm{F}^{\prime}$ region of CIR 04 is shown with blue dots. The temporal evolution of three spectra in the $\mathrm{F}$ region are illustrated with green rectangles and red and cyan triangles. The fitted dashed curves through the observed points are based on the theory of Fisk \& Lee (1980). The turnover shape of the spectra is clearly visible in the fast-wind region of CIR 04.

and $\mathrm{SOHO} / \mathrm{PM}$. Then we varied $\kappa_{0}$ to fit the observed spectrum and obtained $\kappa_{0}=0.077$. For the CIR event studied by Chotoo et al. (2000), they obtained $\kappa_{0}=0.014$, which is smaller than our result, but in a similar order of magnitude. For the three spectra in the F region, we followed the method of Reames et al. (1997) to fit spectra with Eq. (3). $\beta$ and $r_{s}$ were treated as adjustable parameters, and we normalized each spectrum to the observations assuming a fixed $\kappa_{0}$, as $\kappa_{0}$ is considered as a constant in the theory of Fisk \& Lee (1980). For the case of $\kappa_{0}=0.077$, the shock distances $r_{s}$ are derived to be 10, 6.7, and 7.8 AU, and the parameters $\beta$ are $0.40,0.36$, and 0.32 , respectively, corresponding to observed green rectangles and red and cyan triangles. Apparently, the values of $r_{s}$ are much higher than those determined with wind speed data. Although $\beta$ decreases with the connection distance, consistent with the hardening spectra observed by Reames et al. (1997), its values are all lower than those derived at $1 \mathrm{AU}$, indicating a weaker reverse shock beyond $1 \mathrm{AU}$. If we increase the value of $\kappa_{0}$, the above high values of $r_{s}$ can be accommodated, but the derived $\beta$ values will also increase, implying a lower compression ratio beyond $1 \mathrm{AU}$, which is in disagreement with our expectation. Therefore, we suggest that Eq. (3) does not perfectly explain the turnover spectra, and the fitted values of the parameters here should not be taken too literally. Possible reasons for this disagreement are discussed in the next section.

\subsection{Clean events without reverse shocks}

As mentioned by Jian et al. (2006), only about one third of all CIRs at 1 AU have a reverse shock. Nine of the 16 CIR events in our survey have a weak compression region with a gradual pressure decline at the trailing edges. Because we cannot know where reverse shocks form in these events, we cannot list the connection distances of the CIR events shown in Fig. 7. 

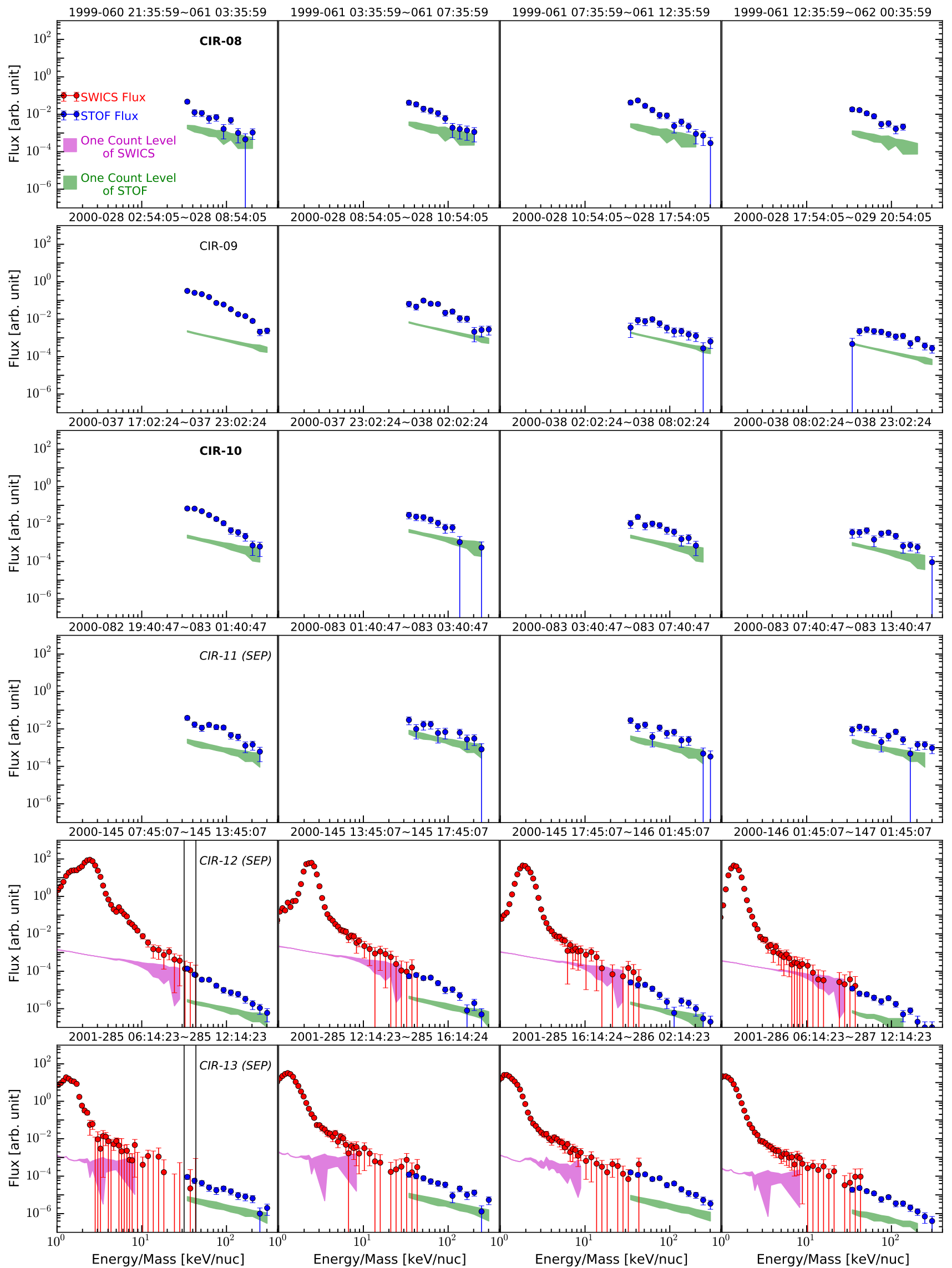

Fig. 7. Same as Fig. 5, but bounded without reverse shocks. 


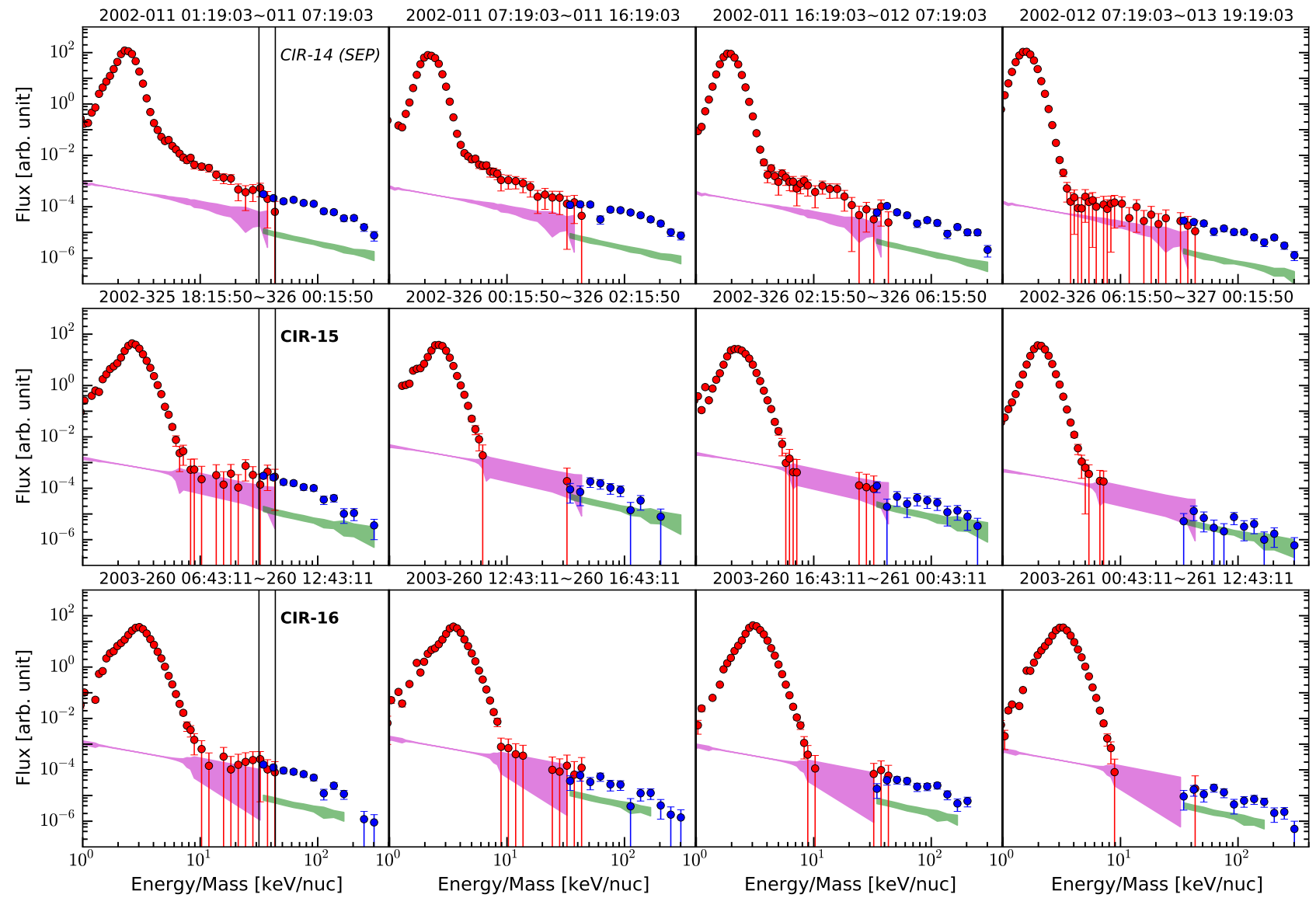

Fig. 7. continued.

Here, we analyse four clean CIR events of the second event group (bottom half of Table 1). In both $\mathrm{F}^{\prime}$ and $\mathrm{F}$ regions, CIR 08 exhibits the usual (power-law) spectral behaviour, which is very similar to the events with shocks (CIRs 02 and 05). Although CIR 10 has only STOF measurements, the statistics in the $\mathrm{F}^{\prime}$ region are good enough to show a turnover shape at the range where SWICS and STOF overlap. Similarly, during CIRs 15 and 16 , turnover parts appear in the energy range $(10-43 \mathrm{keV} / \mathrm{nuc})$ covered by SWICS. In the F region, for the clean CIR 10 event without SWICS data, we note possible turnover spectra. Although unlikely given the shown errors, this may be caused by statistical fluctuations. The cleanest turnover spectrum in this group is shown in the fourth plot of CIR 16 with a flux peak at $\sim 50 \mathrm{keV} /$ nuc. Additionally, we note that in the energy range between the bulk solar wind and STOF measurements in CIR 15, only the very high SWICS $E / q$ channels registered counts in the second and third plots. The same phenomenon also occurs in the third and fourth plots of CIR 16. Although these counts were all registered at the one-count level with large error bars, they are only seen at high energies, and no particles were registered at lower energies by SWICS. It appears reasonable to interpret this observation as a manifestation of a turnover spectrum in the higher energy range. As summarized in Table 1, three of four clean CIR events exhibit possible turnover spectra in both $\mathrm{F}^{\prime}$ and $F$ regions, while the remaining one event shows continuous power-law spectra.

\subsection{Contaminated events}

As observed routinely upstream of the Earth's bow shock, upstream ion events are characterized by a spiky pattern of the intensity time profile of suprathermal particles (Klassen et al. 2008; Klassen et al. 2009), steep power-law spectra $\left(J \propto E^{-4}\right)$ (Desai et al. 2008), and large field-aligned sunward anisotropies (Müller-Mellin et al. 2008). They were mainly found to occur after a CIR passed the Earth's magnetosphere (Kronberg et al. 2011). The duration time for each individual event is mostly less than $10 \mathrm{~min}$ (Klassen et al. 2008). The intensity time profile of CIRs 03 and 09 hint at upstream ion events, and the spectra exhibit power-law suprathermal tails in $\mathrm{F}^{\prime}$ regions. However, we observed a possible superposition of turnover and power-law spectra in the $\mathrm{F}$ region of CIR 03. More specifically, CIR 03 shows a spectral gap in the energy range $\sim 70-100 \mathrm{keV} / \mathrm{nuc}$ in the second plot from the left (see Fig. 5). This may be an indication of a turnover around $100 \mathrm{keV} /$ nuc. Below $\sim 70 \mathrm{keV} / \mathrm{nuc}$, three STOF data points might indicate a power-law tail, which could be the contribution of upstream ion events. Not a single count was observed in the lowest energy bins (32-44 keV/nuc) in the last six hours (rightmost panel). In comparison with CIR 03, spectra of CIR 09 are more like a turnover shape. In the lowest eight energy channels the averaged flux is suppressed more and more heavily as we proceed from the second to the fourth plot, so that it finally falls into the one-count level with the ensuing large (upper limit) error bars, which clearly indicates that the spectrum starts to turn over below $\sim 40 \mathrm{keV} /$ nuc. Hence, the effect from upstream ion events can probably be neglected during CIR 09.

The spectra of four SEP events $(06,12,13$, and 14) in Figs. 5 and 7 show better counting statistics than the other CIR events. All the spectra of these four events exhibit power-law tails in $\mathrm{F}^{\prime}$ regions, while three of them (events $12-14$ ) remain power-law 
spectra in $\mathrm{F}$ regions. The suprathermal spectrum in the $\mathrm{F}$ region of CIR 06 shows a clear power-law spectrum above solar wind energies up to $40 \mathrm{keV} /$ nuc, together with a slight hump just below $\sim 100 \mathrm{keV}$. As we move away from the CIR, this hump persists. However, the signature is not very clear. Event 11 was closely preceded by an impulsive or ${ }^{3} \mathrm{He}$-rich SEP event, and the lowest energy ${ }^{3} \mathrm{He}$ population might still have been present inside this event. On the other hand, this event itself was considered to be an SEP event (Gopalswamy et al. 2002; Cane et al. 2010). The spectrum in the $F^{\prime}$ region of Fig. 7 behaves like a power law with a turnover component, similar to the second plots of events 03 and 06 . However, there is no hint of spectral turnover in three spectra in the F region, but only continuous power-law tails.

\section{Discussion}

In our investigation, 6 of 9 clean CIR events show possible signs of a turnover between $\sim 10-40 \mathrm{keV} /$ nuc, which could be explained by the low-energy threshold of Fisk \& Lee (1980). Mason et al. (1997) investigated as many as 17 CIR events, the energy coverage of their observations is from $\sim 40 \mathrm{keV} /$ nuc to $\sim 6 \mathrm{MeV} /$ nuc, just above the turnover energy range. In the survey by Mason et al. (2008), where 41 CIR events were analysed from 1998 to 2007, the lowest energy of the helium spectra is about $90 \mathrm{keV} / \mathrm{nuc}$, which is also higher than the turnover energy range in this work. Hence, they did not observe turnover spectra.

However, if the Fisk \& Lee (1980) mechanism works, why do not all CIR events show turnover spectra? This is probably due to two reasons: (a) low flux resulting in insufficient counting statistics; and (b) this is the consequence of the admixtures of suprathermal particles from different sources, for example, the $v^{-5}$ tail of the Fisk \& Gloeckler model (Fisk \& Gloeckler 2006, 2007; Fisk et al. 2010), cross-field transport (Baring et al. 1997; Dwyer et al. 1997), upstream ion events, and solar particle events (see in Table 1). As shown in Fig. 2, the flux of suprathermal ions decreases with increasing connection distance, therefore it is difficult to measure the turnover directly because the flux is low, for instance, CIRs 04, 07, 15, and 16. We interpreted the spectral gap as due to the turnover. Additionally, when the flux of remote accelerated particles is low, particles from other sources can become visible in our observations. One of them, the ubiquitous $v^{-5}$ or $E^{-1.5}$ suprathermal tails (Fisk \& Gloeckler 2006, 2007; Fisk et al. 2010), may mask the remotely accelerated suprathermal particles and thus also the turnover. This effect is expected to be more pronounced at CIR reverse shocks than at other shocks because the higher bulk and thermal speed of the fast wind leads to a more efficient injection into the acceleration process (Giacalone \& Jokipii 1997). An additional source of suprathermal particles may be due to enhanced perpendicular (or cross-field) transport associated with CIRs (Baring et al. 1997; Dwyer et al. 1997). In this case we would not necessarily be sampling particles from the reverse shock connected along an ideal Parker spiral, and thus at the inferred connection distance, but from a much closer reverse shock to which we are only connected by some kind of perpendicular diffusion process that is not understood. Another source of suprathermal particles may come from upstream events and solar particle events. For example, events 03, 06, and 11 exhibit a mixture of turnover and power-law spectra in regions $\mathrm{F}$ and $\mathrm{F}^{\prime}$, respectively, while the power-law part might not be of CIR origin.

Although we found several turnover spectra in our investigation, they cannot be well fitted with reliable and physically meaningful parameters based on the theory of Fisk \& Lee (1980). The reason for this disagreement may be the underlying assumption of an isotropic distribution function in the model. In the energy range considered here, this assumption has been shown to be invalid by Ebert et al. (2012). These authors found a large anisotropy in CIR 04. Another possible explanation is that the diffusion coefficient does not necessarily depend linearly on $r$ and $v$, as commonly assumed. For instance, Savopulos et al. (1995) showed that the expression $\kappa=\kappa_{0} v^{\alpha} r^{\epsilon}$ results in a better fit to the spectrum. Furthermore, CIRs are three-dimensional structures, and their magnetic structure is much more complex than theoretical assumptions. Another possible effect of the CIR 3D structure is that ACE and SOHO may not be located in the same flux tube, therefore the combination of data from these two spacecraft shows variable spectra. Despite these shortcomings, we have observed the signature expected for the Fisk \& Lee model in several CIR events, but a different theoretical or numerical approach may be needed to describe the turnover spectra in detail. Ebert et al. (2012) found that the He ion flows in one CIR, which occurred in 2008 and has only a weak compression region, were predominantly sunward streaming in both regions, downstream and upstream of the CIR trailing edge. This agrees with our observation for those CIR events $(10,15$, and 16) with turnover spectra in $\mathrm{F}^{\prime}$ regions, as turnover spectra already indicate that the remote acceleration or sunward particles are dominant. Suprathermal particles observed in the F regions may be attributed to stochastic acceleration in the $\mathrm{F}^{\prime}$ regions beyond $1 \mathrm{AU}$ before the reverse shocks could form. In this case, the Fisk \& Lee model becomes invalid, and the model by Giacalone et al. (2002) may be more appropriate. This model considers the nondiffusive magnetic mirroring effect in the region between the compression and the Sun, where trapped ions can be effectively accelerated by scattering between converging scattering centers. Finally, we also point out that neither SWICS nor STOF directly view the sunward-propagating particles, but only those that are again reflected inside $1 \mathrm{AU}$ and return out to ACE or SOHO. This requires sufficient mirroring inside $1 \mathrm{AU}$ and may add to the obscuration of the Fisk \& Lee (1980) turnover.

\section{Conclusions}

We examined energy spectra of alpha particles from the bulk solar wind $(\sim 1 \mathrm{keV} / \mathrm{nuc})$ to $330 \mathrm{keV} / \mathrm{nuc}$ in 16 events observed between 1999 and 2005. A common feature is that the flux of these low-energy suprathermal particles peaks inside the compressed and decelerated fast-wind region, close to the trailing edges. After the spacecraft crosses the trailing boundary of the CIR, suprathermal particles continue to be observed for typically at least half a day. Away from this boundary, the flux of particles accelerated locally at the reverse shock or pressure wave decreases as they would have to diffuse perpendicularly to the local magnetic field to reach the observer. On the other hand, particles that are remotely accelerated at the reverse shock can travel inward more easily along the magnetic field lines. Our analysis of the suprathermal alpha particles shows that CIRs bounded with a reverse shock at $1 \mathrm{AU}$ have power-law spectra (possibly with an exponential rollover) in the compressed fast solar wind $\left(\mathrm{F}^{\prime}\right)$ region. In the undisturbed fast wind $(\mathrm{F}), 3$ of the 5 clean CIR events bounded by reverse shocks at 1 AU exhibited a possible sign of a turnover in the low suprathermal range, below approximately $40 \mathrm{keV} /$ nuc, which is overall consistent with the basic idea underlying the model of Fisk \& Lee (1980). Nevertheless, it was not possible to fit their model to the data with 
meaningful parameters, suggesting that other effects need to be taken into account. Three of 4 clean CIR events that were not bounded by reverse shocks at $1 \mathrm{AU}$ showed signs of a turnover in both the compressed fast solar wind $\left(\mathrm{F}^{\prime}\right)$ region and fast-wind region $(\mathrm{F})$, indicating that the source of these suprathermal particles lies beyond 1 AU.

Acknowledgements. The authors thank all individuals and institutions who made CELIAS and SOHO possible. We thank the anonymous referee for the helpful and constructive comments, which greatly improved the final version of the paper. We also thank A. Klassen for evaluating the effect of upstream ion events for our measurements. This work and L.B. were supported by grants 50 OC 1103 and 50 OC 1501 from the German Space Agency, DLR. J.Y. thanks the DAAD for financial support, R.F.W.S. acknowledges helpful discussions during the ISSI Team meeting "Exploration of the Inner Heliosphere - what we have learned from Helios and what we want to study with Solar Orbiter".

\section{References}

Balogh, A., Gosling, J., Jokipii, J., Kallenbach, R., \& Kunow, H. 2000, in Corotating interaction regions (Springer Science \& Business Media), 7

Bamert, K., Wimmer-Schweingruber, R. F., Kallenbach, R., et al. 2002, J. Geophys. Res., 107, 1130

Baring, M. G., Ogilvie, W., Ellison, C., \& Forsyth, R. J. 1997, ApJ, 476, 889

Berger, L. 2008, Ph.D. Thesis, Christian-Albrechts-Universität zu Kiel, Germany

Bučík, R., Mall, U., Korth, A., \& Mason, G. M. 2009, Annales Geophysicae, 27, 3677

Cane, H. V., Richardson, I. G., \& von Rosenvinge, T. T. 2010, J. Geophys. Res., 115, A08101

Chotoo, K., Schwadron, N. A., Mason, G. M., et al. 2000, J. Geophys. Res., 105, 23107

Cliver, E. W., Ling, A. G., Belov, A., \& Yashiro, S. 2012, ApJ, 756, L29

Desai, M. I., Mason, G. M., Dwyer, J. R., et al. 2003, ApJ, 588, 1149

Desai, M. I., Mason, G. M., Gold, R. E., et al. 2006, ApJ, 649, 470

Desai, M. I., Mason, G. M., Müller-Mellin, R., et al. 2008, J. Geophys. Res., 113, A08103

Dwyer, J. R., Mason, G. M., Mazur, J. E., et al. 1997, ApJ, 490, L115

Ebert, R. W., Dayeh, M. A., Desai, M. I., \& Mason, G. M. 2012, ApJ, 749, 73

Fisk, L. A., \& Gloeckler, G. 2006, ApJ, 640, L79

Fisk, L. A., \& Gloeckler, G. 2007, Space Sci. Rev., 130, 153

Fisk, L. A., \& Jokipii, J. R. 1999, Space Sci. Rev., 89, 115

Fisk, L. A., \& Lee, M. A. 1980, ApJ, 237, 620

Fisk, L. A., Gloeckler, G., \& Schwadron, N. A. 2010, ApJ, 720, 533

Giacalone, J., \& Jokipii, J. R. 1997, Geophys. Res. Lett., 24, 1723

Giacalone, J., Jokipii, J. R., \& Kóta, J. 2002, ApJ, 573, 845
Gloeckler, G., Cain, J., Ipavich, F. M., et al. 1998, Space Sci. Rev., 86, 497 Gopalswamy, N., Yashiro, S., Michałek, G., et al. 2002, ApJ, 572, L103

Gosling, J. T., Asbridge, J. R., Bame, S. J., \& Feldman, W. C. 1978, J. Geophys. Res., 83, 1401

Hilchenbach, M., Hsieh, K. C., Hovestadt, D., et al. 1998, ApJ, 503, 916

Hilchenbach, M., Grünwaldt, H., Kallenbach, R., et al. 1999, in AIP Conf. Ser. 471, eds. S. R. Habbal, R. Esser, J. V. Hollweg, \& P. A. Isenberg, 605

Hilchenbach, M., Sierks, H., Klecker, B., Bamert, K., \& Kallenbach, R. 2001, International Cosmic Ray Conference, 8, 3144

Hovestadt, D., Hilchenbach, M., Bürgi, A., et al. 1995, Sol. Phys., 162, 441

Ipavich, F. M., Galvin, A. B., Lasley, S. E., et al. 1998, J. Geophys. Res., 103, 17205

Jian, L., Russell, C. T., Luhmann, J. G., \& Skoug, R. M. 2006, Sol. Phys., 239, 337

Klassen, A., Gómez-Herrero, R., Böhm, E., et al. 2008, Annales Geophysicae, 26, 905

Klassen, A., Gómez-Herrero, R., Müller-Mellin, R., et al. 2009, Annales Geophysicae, 27, 2077

Kronberg, E. A., Bučík, R., Haaland, S., et al. 2011, J. Geophys. Res., 116, A02210

Lepri, S. T., \& Zurbuchen, T. H. 2004, J. Geophys. Res., 109, A01112

Lepri, S. T., Zurbuchen, T. H., Fisk, L. A., et al. 2001, J. Geophys. Res., 106, 29231

Mason, G. M., Mazur, J. E., Dwyer, J. R., Reames, D. V., \& von Rosenvinge, T. T. 1997, ApJ, 486, L149

Mason, G. M., Mazur, J. E., Dwyer, J. R., et al. 2004, ApJ, 606, 555

Mason, G. M., Leske, R. A., Desai, M. I., et al. 2008, ApJ, 678, 1458

McComas, D. J., Bame, S. J., Barker, P., et al. 1998, Space Sci. Rev., 86, 563

Morris, D., Möbius, E., Lee, M. A., et al. 2001, in Joint SOHO/ACE workshop "Solar and Galactic Composition", ed. R. F. Wimmer-Schweingruber, AIP Conf. Ser., 598, 201

Müller-Mellin, R., Gomez-Herrero, R., Böttcher, S., et al. 2008, Int. Cosmic Ray Conf., 1, 371

Reames, D. V., Ng, C. K., Mason, G. M., et al. 1997, Geophys. Res. Lett., 24, 2917

Richardson, I. G., \& Cane, H. V. 2010, Sol. Phys., 264, 189

Richardson, I. G., Barbier, L. M., Reames, D. V., \& von Rosenvinge, T. T. 1993, J. Geophys. Res., 98, 13

Savopulos, M., Quenby, J. J., \& Bell, A. R. 1995, Sol. Phys., 157, 349

Schwadron, N. A., Fisk, L. A., \& Gloeckler, G. 1996, Geophys. Res. Lett., 23, 2871

Simnett, G., Kunow, H., Flückiger, E., et al. 1998, Space Sci. Rev., 83, 215

Smith, C. W., L'Heureux, J., Ness, N. F., et al. 1998, Space Sci. Rev., 86, 613

Wimmer-Schweingruber, R. F., von Steiger, R., \& Paerli, R. 1997, J. Geophys. Res., 102, 17407

Wimmer-Schweingruber, R. F., von Steiger, R., \& Paerli, R. 1999, J. Geophys. Res., 104, 9933

Yu, J., Berger, L., Wimmer-Schweingruber, R. F., et al. 2016, AIP Conf. Proc., 1720 